\title{
Velocity measurement accuracy in optical microhemodynamics: experiment and simulation
}

\author{
Boris Chayer ${ }^{1}$, Katie L Pitts ${ }^{2}$, Guy Cloutier ${ }^{1,3}$ and Marianne Fenech ${ }^{2,4,5}$ \\ ${ }^{1}$ Laboratory of Biorheology and Medical Ultrasonics, University of Montreal Hospital Research \\ Center (CRCHUM), Montreal, Quebec, Canada \\ 2 Department of Chemical and Biological Engineering, University of Ottawa, Ottawa, Ontario, \\ Canada \\ ${ }^{3}$ Department of Radiology, Radio-Oncology and Nuclear Medicine, Institute of Biomedical \\ Engineering, University of Montreal, Montreal, Quebec, Canada \\ ${ }^{4}$ Department of Mechanical Engineering, University of Ottawa, Ottawa, Ontario, Canada \\ E-mail: mfenech@uottawa.ca
}

Received 7 December 2011, accepted for publication 18 July 2012

Published 4 September 2012

Online at stacks.iop.org/PM/33/1585

\begin{abstract}
Micro particle image velocimetry ( $\mu$ PIV) is a common method to assess flow behavior in blood microvessels in vitro as well as in vivo. The use of red blood cells (RBCs) as tracer particles, as generally considered in vivo, creates a large depth of correlation (DOC), even as large as the vessel itself, which decreases the accuracy of the method. The limitations of $\mu \mathrm{PIV}$ for blood flow measurements based on RBC tracking still have to be evaluated. In this study, in vitro and in silico models were used to understand the effect of the DOC on blood flow measurements using $\mu$ PIV RBC tracer particles. We therefore employed a $\mu$ PIV technique to assess blood flow in a $15 \mu \mathrm{m}$ radius glass tube with a high-speed CMOS camera. The tube was perfused with a sample of $40 \%$ hematocrit blood. The flow measured by a cross-correlating speckle tracking technique was compared to the flow rate of the pump. In addition, a threedimensional mechanical RBC-flow model was used to simulate optical moving speckle at $20 \%$ and $40 \%$ hematocrits, in 15 and $20 \mu \mathrm{m}$ radius circular tubes, at different focus planes, flow rates and for various velocity profile shapes. The velocity profiles extracted from the simulated pictures were compared with good agreement with the corresponding velocity profiles implemented in the mechanical model. The flow rates from both the in vitro flow phantom and the mathematical model were accurately measured with less than $10 \%$ errors. Simulation results demonstrated that the hematocrit (paired $t$ tests, $p=0.5$ ) and the tube radius $(p=0.1)$ do not influence the precision of the measured flow rate, whereas the shape of the velocity profile $(p<0.001)$ and the location of the focus plane $(p<0.001)$ do, as indicated by measured errors ranging from $3 \%$ to $97 \%$. In conclusion, the use of RBCs as tracer particles makes a large
\end{abstract}

5 Author to whom any correspondence should be addressed. 
DOC and affects the image processing required to estimate the flow velocities. We found that the current $\mu$ PIV method is acceptable to estimate the flow rate on the condition that the measurement takes place at the equatorial plane of the vessel. Otherwise, it is not an appropriate method to estimate the shape of the velocity profile.

Keywords: blood, red blood cell, microcirculation, velocity profile, microparticle image velicometry, cross-correlation, depth of correlation

(Some figures may appear in colour only in the online journal)

\section{Nomenclature}

$\mu \mathrm{PIV}$ microscopic particle image velocimetry

2D two-dimensional

3D three-dimensional

a radius of a particle

CC cross-correlation

DOC depth of correlation

$\mathrm{D}_{a} \quad$ lens aperture diameter

$d_{e} \quad$ effective diameter of an out-of-focus RBC (due to shadowing)

$d_{i j} \quad$ distance between two centers of mass of RBCs $i$ and $j$

$d_{p} \quad$ particle diameter

$H$ hematocrit (volume fraction of RBCs in blood)

$f_{i}^{h} \quad$ hydrodynamic force acting on particle $i$

$f_{i j}^{e} \quad$ force of the particle $j$ acting on particle $i$

$f_{i w}^{e} \quad$ the force induced by the wall $W$ on $i$

$J_{p} \quad$ flux of light from the particle

$k \quad$ velocity profile shape coefficient

$K_{E} \quad$ elasticity coefficient

$M \quad$ magnification

$\mathrm{m}_{i} \quad$ mass of the particle $i$

MVP temporal mean velocity of a particle

$n \quad$ index of refraction of the immersion medium between the microfluid and the lens

NA the numerical aperture of the lens

$\boldsymbol{n}_{i j} \quad$ the normalized vector pointing from $\operatorname{RBC} j$ to $i$.

$p \quad p$-value from paired $t$ test

$Q \quad$ volume flow rate

$R \quad$ vessel radius

$\mathrm{RBC}$ red blood cell

ive used for estimation from the in vitro experiment

ose used for estimation from the optical simulation

ms used for mechanical simulations

$p \quad$ used for pump

$\boldsymbol{v}_{i} \quad$ velocity of the particle $i$

$V_{\max }$ maximum velocity

$\beta \quad$ parameter of the Gaussian distribution of the light 
$\lambda \quad$ wavelength of light

$\mu \quad$ dynamic viscosity of the fluid

$\varepsilon \quad$ distance from the object plane beyond which the contribution becomes insignificant

\section{Introduction}

The medical and fluid mechanics communities have become increasingly interested in properties of blood flow in the microcirculation. The microcirculation, which is composed of numerous vessels with diameters typically less than $50 \mu \mathrm{m}$, is the main exchange site between blood and tissues. Because blood behavior in the microcirculation cannot be considered as a liquid flow, but rather as a collection of interacting particles in suspension, the study of blood flow in the microcirculation represents a considerable challenge. The better understanding of the characteristics of blood flow in the microcirculation is of interest to understand mechanical interactions between blood components and the vessel wall, consequently giving insight into the causes of cardiovascular diseases, cardiogenesis and angiogenesis.

Micro-particle image velocimetry ( $\mu$ PIV) has been recently used to determine the blood velocity field and consequently the instantaneous velocity profiles of blood flowing through micro-channels. Generally, for in vivo studies (i.e. for measuring the blood velocity profile in a microcirculation bed on a living subject) red blood cells (RBCs) are used as tracer particles for the $\mu$ PIV method (Bitsch et al 2005, Kikuchi and Mochizuki 2011, Koutsiaris and Pogiatzi 2004, Lee et al 2007, Sugii et al 2002, Tsukada et al 2000); while for in vitro studies, artificial micro fluorescent particles are usually added to blood (Lima et al 2006, 2007, 2008, Sugii et al 2005). Note that a few studies showed the possibility of using artificial tracer particles in living subjects; see for examples results in an embryonic heart (Poelma et al 2010, Vennemann et al 2006) or in the chicken vitelline network (Poelma et al 2008). The use of RBCs has the advantage of not requiring particle injection and, more importantly, it avoids confounding interpretation attributed to the modification of the system under study. Thus, it is a method of high interest for dynamic video microscopy of the human microcirculatory flow. The use of RBCs as imaging tracers has nevertheless some disadvantages because their diameter is approximately $7 \mu \mathrm{m}$, while the diameter of an artificial fluorescent particle is typically on the order of $1 \mu \mathrm{m}$. The higher RBC particle size and its density are responsible for the decrease in accuracy of the measurement method (Nguyen et al 2010, Kloosterman et al 2011).

The goal of this study was thus to assess the intrinsic error of the measurement method when using RBCs as tracer particles. In this study, in vitro and in silico models were used to assess the accuracy of the flow rate and velocity profile estimations. The in vitro model allowed for a highly controlled pump flow that could be compared to the $\mu$ PIV video microscopy measurements. In order to further validate the instantaneous blood flow velocity profiles obtained with the imaging technique, a computational model of the microscopy image formation was developed. The synthetic images of moving RBCs were built based on a mechanical model mimicking three-dimensional (3D) flow in a circular channel.

\section{Theoretical background}

The cross-correlation (CC) between two consecutive optical images is a standard method used to calculate particle velocities. An interrogation window in the first image is compared within an interrogation window grid in the second image to find the most look-alike windows. The distance between the center of both windows can be divided by the time elapsed between 


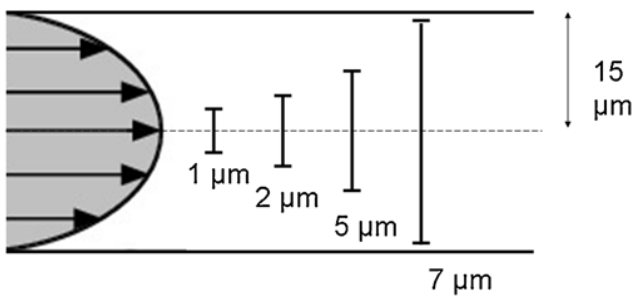

Figure 1. Theoretical values for the depth-of-correlation (DOC) relative to $15 \mu \mathrm{m}$ radius capillary for different size of tracer particle.

each frame to calculate the mean velocity of particles for this position. Even if the $\mathrm{CC}$ is accepted as a flexible and robust method (Cheezum et al 2001), this algorithm needs to be validated for applications dealing with flowing cells, as in the case of microcirculatory blood flow. More specifically, it is important to understand the effect of out-of-focus particles and resulting image blurring on the estimation of velocities. The depth of correlation (DOC) is a concept in microscopy that takes into account the manner in which the image is built and used (Meinhart et al 2000). It describes how far one particle, over or under the focal plane, affects image-processing algorithms and can be interpreted as a measurement of the spatial resolution in the $Z$ dimension.

To measure velocities only of particles in the focal plane, as in $\mu$ PIV with seeding particles at a low volume concentration, some recommendations were made. Indeed, the particle volume concentration should be less than $0.1 \%$ and the particle diameter should be below $1 \mu \mathrm{m}$ (Meinhart et al 2000, Olsen and Adrian 2000). These guidelines are not applicable to blood flow, because the largest RBC diameter is approximately $7 \mu \mathrm{m}$ and the hematocrit (volume concentration) is between $20 \%$ (in the microcirculation) and $45 \%$ (in the systemic circulation), approximately. Consequently, all RBCs typically present in a microvessel with a $15 \mu \mathrm{m}$ radius may affect image processing algorithms; measured velocities could then be biased because they correspond to a weighted average of cell velocities both in and out the focal plane.

To describe this phenomenon, the DOC was defined as the minimal distance that separates a particle from the image plane without affecting the image processing method (Wereley et al 1998):

$$
\mathrm{DOC}=\frac{1}{2}\left[\frac{(1-\sqrt{\varepsilon})}{\sqrt{\varepsilon}}\left(\frac{n^{2}}{\mathrm{NA}^{2}}-1\right)\left(d_{p}^{2}+\frac{1.49(M+1)^{2} \lambda^{2}}{M^{2} \mathrm{NA}^{2}}\right)\right]^{1 / 2}
$$

where $\varepsilon$ is the minimal intensity of an away particle affecting the CC (we can empirically set $\varepsilon$ to a value of $10 \%$ of the intensity of an in-focus particle) (Meinhart et al 2000), $n$ is the index of refraction of the immersion medium between the microfluid and the lens $(n=1.33$ for water), $\lambda=0.6 \mu \mathrm{m}$ is the wavelength of light, NA is the numerical aperture of the lens, $d_{p}$ is the particle diameter, and $M$ is the magnification.

Equation (1) gives a DOC of $14 \mu \mathrm{m}$ for a $\mathrm{RBC}$ with $d_{p}=7 \mu \mathrm{m}$, when it is observed with a lens characterized by $M=40 \times$ and NA $=0.8$. This confirms that all RBCs present in a typical microvessel of $15 \mu \mathrm{m}$ radius are visible and can affect the assessment of velocity profiles. Figure 1 shows the theoretical values for the DOC relative to the $15 \mu \mathrm{m}$ radius capillary for different sizes of tracer particles. As shown later, the effect of using RBCs as tracer particles was highlighted both experimentally and numerically by forming synthetic images of blood flow in the microcirculation. 


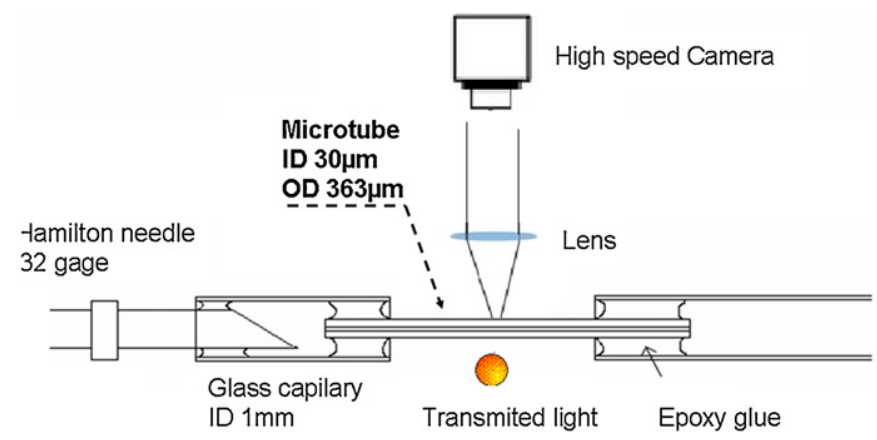

Figure 2. Schematic of the microcirculation in vitro set-up with glass tubing having a $15 \mu \mathrm{m}$ radius.

\section{Materials and method}

\subsection{Experimental model}

3.1.1. In vitro model. An in vitro model of the microcirculation was built with fused silica tubing (no. 062801, SGE Inc, Austin, TX, USA) having a radius of $15 \mu \mathrm{m}$ and an outer diameter of $363 \mu \mathrm{m}$, glued with epoxy in $1 \mathrm{~mm}$ inner diameter glass capillaries at both ends (figure 2). The geometry (round channel) and scale (15 $\mu$ m radius) used here position this in vitro study as the closest to the physiological measurements currently being made in intravital microscopy. A microsyringe connected to a 32-gauge needle (no. 80016, Hamilton, Reno, NV, USA) was glued at one end of the model to allow perfusion of the microtube with minimal dead volume between it and the needle. The syringe was fixed on a computer-controlled syringe pump to inject blood into the microcirculation model. Blood flow images were acquired with an Axiotech Vario microscope (Zeiss, Oberkochen, Germany) equipped with a $40 \times$ water immersion lens $(\mathrm{NA}=0.8)$ in transmission light mode. A Zeiss, KL 1500 LCD cold light source connected to flexible optic fiber was used to improve the illumination of the in vitro model. In addition, a cold filter cutting the red component of the lighting and increasing the contrast between the RBC and the background was added. A $0.63 \times \mathrm{C}$-mount adaptor allowed the recording of image sequences via a high-speed digital camera (no. 1M150, Dalsa, Waterloo, Ontario, Canada). The camera connected to a digital frame grabber (Hélios, Matrox, Montreal, Quebec, Canada) recorded 50 frames at a frame rate of $2000 \mathrm{~s}^{-1}$ at a resolution of $120 \times$ 170 pixels with a calibrated isotropic pixel width of $0.42 \mu \mathrm{m}$. Image sequences were postprocessed with a 2D CC algorithm implemented in Matlab (ver. 7 R14, Natick, MA, USA).

3.1.2. Experiments. Whole porcine blood was used. Blood samples were collected from a slaughterhouse and mixed with $3 \mathrm{~g}$ of EDTA per liter of blood. The blood was centrifuged and RBCs were washed twice with saline to remove the white blood cells and platelets, and RBCs were then re-suspended at $40 \%$ hematocrit in the native plasma. Platelet and white blood cells were removed as it was assumed they do not affect the image formation as their concentrations are negligible regarding the concentration of $\mathrm{RBC}$ and thus their participation in the image formation are negligible. The RBCs were re-suspended in saline to avoid aggregation in order to eliminate this confounding factor. First, aggregation increases the variability between samples. Second, aggregation is well known to make the velocity profile flatter at low shear increasing the viscosity and the non-Newtonian behavior. The interpretation of the shape of the velocity profile would then be difficult to attribute either to the aggregating characteristic of 


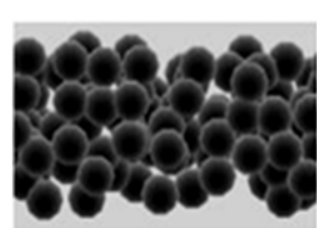

(a)

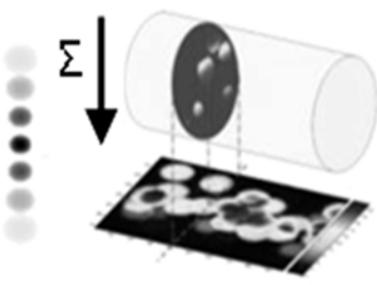

(b)

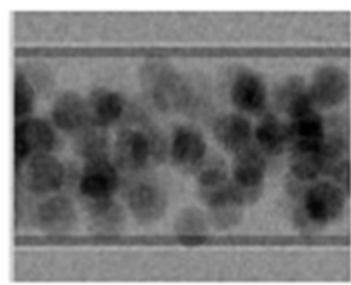

(d)

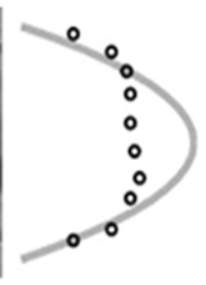

(e)

Figure 3. (a) 3D RBC flow from the mechanical model, (b) particle light and size depending on position, (c) image formation, (d) a synthetic image obtained and (e) dots representing the MVP of $\mathrm{RBC}$ from a time series of synthetic images with the $\mathrm{CC}$ algorithm. The full line in (e) represents the true velocity profile of RBCs imposed in the mechanical model in (a).

the sample or to the measurement method. Note that the mechanical model takes into account the possible flat shape of the velocity profile. Flow measure series varied from 1 to $3 \mu \mathrm{L}$ $\mathrm{h}^{-1}$ (using a $5 \mu \mathrm{L}$ syringe) or $8 \mu \mathrm{L} \mathrm{h}^{-1}$ (using a $10 \mu \mathrm{L}$ syringe) (Tuma et al 2008) at a fixed focus plane in the middle of the tube. These flow rates are based on physiological velocities in the microcirculation from 0.2 to $2.4 \mathrm{~mm} \mathrm{~s}^{-1}$ in venules with a diameter from 18 to $72 \mu \mathrm{m}$ (Popel and Johnson 2005). Forty-five seconds were allowed to elapse between each flow step to obtain a steady flow in the model. A $40 \mu \mathrm{m}$ range of focus, including the tube center plane, was investigated by changing the focal plane in $2 \mu \mathrm{m}$ steps above and below the central plane. The effect of changing the focal plane was evaluated at constant flow rates of 1.5 and $8 \mu \mathrm{L} \mathrm{h}^{-1}$. Each measurement for different flows and focal planes was repeated nine times and averaged; each of them was realized with: three blood samples from different pigs and repeated three times each. The center of the tube was defined as the location where the velocity is maximum, as done by Kloosterman et al (2011).

\subsection{Synthetic image of blood}

A computational model of the microscopy image formation was developed. This section presents the mechanical model that was used to mimic three-dimensional (3D) RBC movements in a tubular parabolic flow, the strategy adopted to produce synthetic images and the $\mathrm{CC}$ algorithm used to measure velocity profiles. Figure 3 summarizes these different steps.

3.2.1. Mechanical modeling of $3 D$ RBC motions. Blood was considered as a collection of spheres interacting with each other and with the vascular wall. The mechanical 2D modeling in Fenech et al (2009) was extended to 3D to mimic real RBC movements in a tubular parabolic flow. The acceleration of a particle $i$ over time $t$ was given by:

$$
m_{i} \frac{\mathrm{d} \boldsymbol{v}_{i}}{\mathrm{~d} t}=\sum_{i \neq j} \boldsymbol{f}_{i j}^{e}+\boldsymbol{f}_{i w}^{e}+\boldsymbol{f}_{i}^{h}
$$

where $m_{i}$ is the mass of the particle $i, \boldsymbol{v}_{i}$ is its velocity, $\boldsymbol{f}_{i j}^{e}$ represents the force of the particle $j$ acting on particle $i, f_{i w}^{e}$ is the force induced by the vascular wall $W$ on $i$, and $f_{i}^{h}$ is the hydrodynamic force. The hydrodynamic force $f_{i}^{h}$ induced by the Stokes drag on a sphere, assuming spherical isolated solid particles in a Newtonian fluid (here the plasma), with very small Reynolds and Stokes numbers (respectively $<1$ and $<0.005$ ), was given by:

$$
\boldsymbol{f}_{i}^{h}=6 \pi \mu a\left(v_{i}^{0} \mathbf{e}_{i}^{0}-\boldsymbol{v}_{i}\right)
$$


where $\mu$ is the dynamic viscosity of the fluid, $a$ is the radius of the particle, $\boldsymbol{v}_{i}$ is the particle velocity at a given time, and $v_{i}^{0} \mathbf{e}_{i}^{0}$ denotes the velocity of the fluid in the absence of particles, which drives the particle $i$ to flow along the direction $\mathbf{e}_{i}^{0}$ at a speed of $v_{i}^{0}$. To simulate a parabolic or a flat velocity profile, the drive velocity was defined as follows:

$$
v_{i}^{0} \mathbf{e}_{i}^{0}=V_{\max }\left[1-\left(\frac{r}{R}\right)^{k}\right] x
$$

where $\boldsymbol{x}$ is the flow direction, $r$ is the radial coordinate, $R$ is the radius of the vessel, and $V_{\max }$ is the maximum centerline velocity. Note that $k=2$ defines a parabolic profile and $k>2$ a blunted profile. The elastic force was to exempt overlapping of RBCs. This force was inspired by a granular interaction model (Duran 1997) and is given by:

$$
\begin{aligned}
\boldsymbol{f}_{i j}^{e} & =K_{E}\left(2 R-d_{i j}\right)^{3 / 2} \boldsymbol{n}_{i j} & & \text { if } d_{i j}<2 a \\
& =0 & & \text { otherwise }
\end{aligned}
$$

where $K_{E}$ is the elasticity coefficient, $d_{i j}$ represents the distance between centers of mass of RBCs $i$ and $j$, and $\boldsymbol{n}_{i j}$ is the normalized vector pointing from $\mathrm{RBC} j$ to $i$. Equation (5) shows that this force tends to repel RBCs when the distance between their centers of mass is smaller than the cell diameter. In the same way, the force between particle $i$ and the wall was calculated as:

$$
\begin{aligned}
\boldsymbol{f}_{i w}^{e} & =K_{E}\left(R-d_{i w}\right)^{3 / 2} \boldsymbol{n}_{i j} & & \text { if } d_{i w}<a \\
& =0 & & \text { otherwise. }
\end{aligned}
$$

RBCs were modeled as spheres of radius $a=2.8 \mu \mathrm{m}$ to mimic the true volume of an erythrocyte, and their mass $m$ was set to $9.8 \times 10^{-14} \mathrm{~kg}$. Particles were initially positioned randomly without overlap in the 3D space. With the use of a finite difference first order scheme, the velocities and positions of particles at time $t+\mathrm{d} t$ were deduced from the acceleration at instant $t$ according to equation (2). The time step was adapted at each iteration to obtain a maximal displacement of $0.05 \mu \mathrm{m}$ for all RBCs. This method offered a good compromise between the stability of the numerical results and the computation time.

3.2.2. Synthetic images. Mechanical simulations gave the positions of RBCs as a function of time. From these positions at each time step (2000 images per second as in the experimental part), we simulated a 2D magnified image using microscopy image formation equations that consider shadowing and illumination effects (Fenech et al 2008).

Shadowing effect. The effective diameter $\left(d_{e}\right)$ of an out-of-focus RBC (due to shadowing) shifted a distance $z$ from the object plane was approximated by (Olsen and Adrian 2000):

$$
d_{e}(z)=\left[M^{2} d_{p}^{2} \frac{1.49(M+1)^{2} \lambda^{2}}{M^{2} \mathrm{NA}^{2}}+\frac{M^{2} D_{a}^{2} z^{2}}{\left(s_{0}^{2}+z\right)^{2}}\right]
$$

where the magnification $M=40 \times$, the numerical aperture of the lens NA $=0.8$, the wavelength of light emitted by the particles is $\lambda=0.6 \mu \mathrm{m}$ (red), the particle diameter $d_{p}=$ $5.6 \mu \mathrm{m}$, the working distance $s_{0}=3.6 \mathrm{~mm}$, and the lens aperture diameter $D_{a}=s_{0} /(2 \times \mathrm{NA})$.

Illumination effect. The total light flux from a single voxel was modeled as 0 if in an empty zone and as $I(z) / N v$ if the voxel was located in an RBC. $N v$ is the number of voxels that discretized the RBC and $I(z)$ is given by equation (8) (Olsen and Adrian 2000):

$$
I(z)=\frac{J_{p} D_{a}^{2} \beta^{2}}{4 \pi d_{e}^{2}\left(s_{0}+z\right)^{2}} \exp \left(\frac{-4 \beta^{2} a^{2}}{d_{e}^{2}}\right)
$$


where $J_{p}$ is the flux of light from the particle that was normalized to $1, \beta=\sqrt{3.67}$ is the parameter of the Gaussian distribution of the light intensity (Olsen and Adrian 2000), and $a=$ $d_{p} / 2$ is the RBC radius. The 3D space was discretized in voxels of $0.42 \times 0.42 \times 0.42 \mu \mathrm{m}^{3}$ to fit images obtained with the experimental set-up. The 2D image was obtained by summing up the weights of all voxels following the direction $z$. Finally, the matrix was normalized by applying a linear transformation to have the same maximum intensity as in the experimental pictures, and, as CMOS senser noise can blur the contrast information in the picture, a noise level equivalent to that of the actual digital camera was added by superposition of a real 'white' picture taken with our camera.

3.2.3. Simulation parameters. In the following, the velocities obtained from mechanical simulations based on equations (2)-(6) are designated with the superscript 'ms' (for mechanical simulations) and velocities estimated with the CC method and computed from simulated optical images (equations (7) and (8)) are labeled with the superscript 'ose' (for estimation from optical simulations). RBC displacements were simulated for hematocrits $(H)$ of $20 \%$ and $40 \%$, in vessels of 15 and $20 \mu \mathrm{m}$ radii $(R)$. To consider a range of flowing conditions, three shapes of velocity profile were implemented from sharp to flat as shown by Bishop et al (2001a) who found $k$ from 1.5 to 4 as a function of the aggregation level of moving RBCs. Therefore, a sharp velocity profile $(k=1.5)$, a parabolic profile $(k=2)$ and a blunted profile $(k=3)$ were implemented. For each case, the maximum velocity $V_{\max }$ was incremented, using 20 steps of $0.1 \mathrm{~mm} \mathrm{~s}^{-1}$, from $0.1 \mathrm{~mm} \mathrm{~s}^{-1}$ to $2 \mathrm{~mm} \mathrm{~s}^{-1}$, which correspond to about 0.1 to $2.5 \mu \mathrm{L} \mathrm{h}^{-1}$ (it could vary depending on the shape of the velocity profile and the tube diameter). Note that velocities above $1 \mathrm{~mm} \mathrm{~s}^{-1}$ could not be reached for the $20 \mu \mathrm{m}$ radius with a $40 \%$ hematocrit on account of processor capability. The synthetic images were made for seven focus planes, labeled $F=0$ to $F=30$, where $F$ indicates the distance in the $z$ direction between the focus plane and the center of the vessel. The different combinations of these parameters generated 1428 movies. Velocity profiles were fitted using equation (4) to determine maximum velocities $V^{\mathrm{ms}}{ }_{\max }$ and $V^{\mathrm{ose}}{ }_{\max }$ and shape parameters $k^{\mathrm{ms}}$ and $k^{\mathrm{ose}}$. Note that $V^{\mathrm{ms}}{ }_{\max }$ and $k^{\mathrm{ms}}$ could be slightly different (less than 5\%) from $V_{\max }$ and $k$ in equation (4) since the mechanical model accounts for RBC interactions due to collisions between neighboring cells. The flow rates $Q^{\mathrm{ms}}$ and $Q^{\text {ose }}$ were computed from $V^{\mathrm{ms}}{ }_{\max }, k^{\mathrm{ms}}$ or $k^{\text {ose }}, V^{\text {ose }}{ }_{\max }$ using equation (10).

\subsection{Computation of velocity profiles with the cross-correlation method}

The same algorithm was used to extract the velocity for both experimental and synthetic images. Due to the very low RBC speed with regard to the camera simulated frame rate, the $\mathrm{CC}$ was computed on frames 1 and 4,2 and 5, and so on, in a manner that increases the time between images to $0.002 \mathrm{~s}$. RBCs at a physiological hematocrit are not distinguishable on microscopic experimental images; thus, the speckle size was used to ascertain the dimension of correlation windows. That size was set by an empirical method that allowed the determination of particle displacements for the range of flow rates considered in this study. Square windows were chosen instead of rectangular ones to facilitate the eventual application of the algorithm on tortuous real vessels, therefore it will not be necessary to rotate the images or the windows in regard of the vessel direction. The size of the first correlation window was set to $15 \times$ 15 pixels $\left(6.3 \times 6.3 \mu \mathrm{m}^{2}\right)$, the size of the second interrogation window to $65 \times 65$ pixels $\left(27.3 \times 27.3 \mu \mathrm{m}^{2}\right)$. An overlap of $100 \%$ minus one pixel was chosen to get a high density of velocity vectors ( 71 speed vectors for the width of the glass microvessel) because the oftenused sampling with $50 \%$ overlap would only provide 11 speed vectors. Oversampling was helpful in this study, as it allowed narrow-band filtering to decimate spurious vectors created 
by noisy images. A median filter was employed to reject all vectors with a modulus or angle different from the median value plus one standard deviation computed from all vectors on the same stream line. In addition, a mean filter was used on 50 successive images based on the hypothesis that, for a given point in an image, steady velocity rates were achieved by the use of the high precision syringe pump (having a resolution of $0.082 \mu \mathrm{m} / \mathrm{step}$ ). By using both filtering strategies (median filter and time mean filter), a mean velocity profile (MVP) was determined for each video sequence, for a flow rate of $1.5 \mu \mathrm{L} \mathrm{h}^{-1}$, a hematocrit of $40 \%$ and acquisitions at the center of the tube (Kloosterman et al 2011).

\subsection{Estimation of blood flow}

3.4.1. Experimentally. To validate the precision of the $\mathrm{CC}$ algorithm and to assess its sensitivity to out-of-focus particles, estimated flows were compared with flows of the pump $Q^{p}$ for a wide range of blood flows and focal planes within the tube. The MVP represents the temporal mean speed of particles, and with equation (9), it allowed the estimation of the flow rate $Q^{\text {ive }}$ (for estimation from in vitro).

$$
Q^{\mathrm{ive}}=\pi \int_{-R}^{R} \text { MVP.r.d } r .
$$

As the MVP extended out of the microtube because of the relatively large correlation windows of our microscopic images, velocity profiles were limited to the dimension of the tube to reduce errors in flow estimation during the integration.

3.4.2. From simulations. Both parameters $V_{\max }$ and $k$ obtained from the best fit of equation (4) were used to compute the flow rate by integrating the velocity profile as:

$$
Q=V_{\max } \pi R^{2} \frac{k}{k+2}
$$

\section{Results}

In this section, the experimental and synthetic results are compared, the parameters that generate an error in the estimation of the flow are determined, and estimation errors of the flow rate are quantified. Finally, estimated shapes of velocity profiles are evaluated.

\subsection{Flow rate estimation with the CC method}

4.1.1. In silico and in vitro flow rate comparison. Figure 4 presents in vitro flow rate estimations with the $\mathrm{CC}$ method versus those of the syringe pump for a $15 \mu \mathrm{m}$ radius glass tube at $H=40 \%$ for the focus in the middle of the vessel $(F=0)$. The linear relation between the estimated flow and the pump flow can be seen by the linear regression (long dashed line) $Q^{\text {ive }}=0.91 Q^{p}$ with $R$-squared equal to 0.95 . The error bars show the standard deviations for nine experiments ${ }^{6}$. The standard deviations are quite large because blood samples from different pigs behaved differently. Figure 4 also shows in silico data for different hematocrits $(\mathrm{H} 20 \%$ and $\mathrm{H} 40 \%)$ and $k(1.5,2$ and 3$)$ in the $15 \mu \mathrm{m}$ radius vessel. The in silico data linear regression (small dashed line) corresponds to $Q^{\text {ose }}=0.90 Q^{\mathrm{ms}}$ with $R$-squared equal to 0.91 . In vitro and in silico linear regressions are well matching. Both experimental and simulated flow rate assessments show an underestimation with respect to the true flow rate of less than $10 \%$.

6 Individual data differing from the average by more than 2 SD have been discarded. 


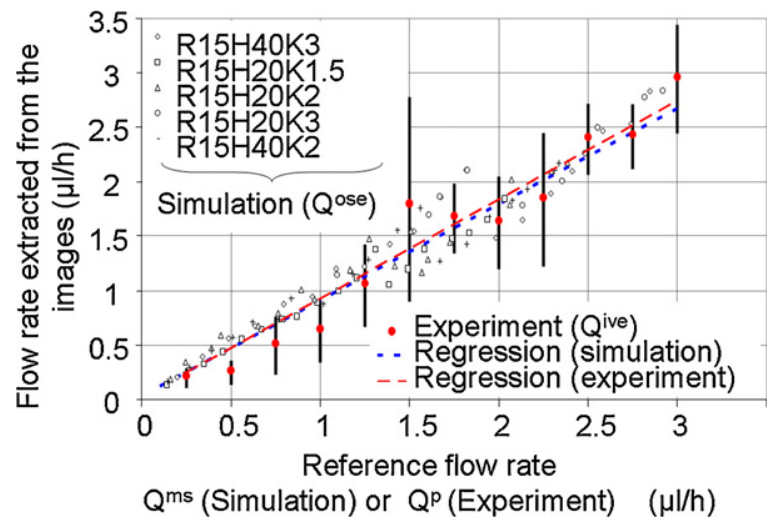

Figure 4. Estimated flow rate by $\mathrm{CC}$ analysis from the in vitro and in silico data as a function of the flow rate of the pump or implemented in the mechanical 3D model for a $15 \mu \mathrm{m}$ radius vessel with an hematocrit of $40 \%$ and $20 \%$ and a velocity profile with $k=1.5,2$, and 3 .

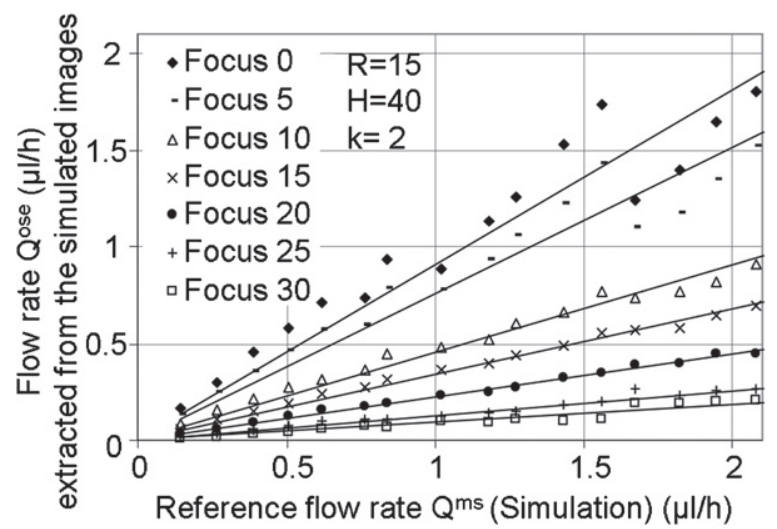

Figure 5. Estimated flow rate by $\mathrm{CC}$ analysis from the synthetic data as a function of the flow rate implemented in the mechanical 3D model for a $15 \mu \mathrm{m}$ radius vessel with a hematocrit of $40 \%$ and a parabolic profile $(k=2)$. Results for different positions of the focus plane (numbers represent the distance of the focus plane from the center of the tube).

4.1.2. In silico estimated flow rate with the CC method versus the flow rate of the mechanical simulation model. For the 1428 in silico sequences, corresponding to the parameters $(R, H$, $k, F, V)$, the flow was calculated by the $\mathrm{CC}$ method. Then for each combination $(R, H, k, F)$, the estimated CC flow was plotted as a function of the flow imposed in the mechanical model. Regarding the synthetic data, for each of the 84 combinations $(R, H, k, F)$, a linear relationship was obtained, where $R$-squared was found always higher than 0.9 except for $(\mathrm{R} 15, \mathrm{H} 20, k 3$, F5) where $R$-squared was 0.81 . Figure 5 presents examples of these linear relationships for different positions of the focus in a $15 \mu \mathrm{m}$ radius vessel at $40 \%$ hematocrit and an imposed parabolic profile $(k=2)$. In this figure, $\left(1-Q^{\text {ose }} / Q^{\mathrm{ms}}\right) \times 100$ (with $Q^{\text {ose }} / Q^{\mathrm{ms}}$ the estimated flow rate divided by the gold standard flow rate $Q^{\mathrm{ms}}$ of the mechanical model) quantifies the error of the $\mathrm{CC}$ method with regard to the theoretical blood flow. The relative errors were found between $3 \%$ and $97 \%$ depending mostly on the parameters $k$ and $F$, as stated in subsection 4.2. Table 1 presents the ratio $Q^{\text {ose }} / Q^{\mathrm{ms}}$ for the focus at 0 (i.e. at the center of the simulated tube). At this position, the minimum and maximum errors were 3 and $35 \%$, respectively. 
Table 1. In silico ratio of $Q^{c} / Q^{m}$ obtained by correlation of the flow rate from 0 to $2 \mu \mathrm{L} \mathrm{h}^{-1}$ (correlation coefficient $R^{2}$ in brackets). Hematocrit $20 \%$ and $40 \%$, radii 15 and $20 \mu \mathrm{m}$ and different velocity profile shape factors $(k=1.5,2$ and 3). Focal plane position is in the middle of the tube $(F=0)$. Figure 6 gives examples of the correlation for $R=20, H=40$ and $k=2$.

\begin{tabular}{lccccc}
\hline & \multicolumn{2}{c}{$\mathrm{R} 15$} & & \multicolumn{2}{c}{$\mathrm{R} 20$} \\
\cline { 2 - 3 } \cline { 5 - 6 }$k$ & $\mathrm{H} 20$ & $\mathrm{H} 40$ & & $\mathrm{H} 20$ & $\mathrm{H} 40$ \\
\hline 1.5 & $0.88\left(R^{2}=0.97\right)$ & $0.65\left(R^{2}=0.90\right)$ & & $0.79\left(R^{2}=0.98\right)$ & $0.79\left(R^{2}=0.99\right)$ \\
2 & $0.92\left(R^{2}=0.93\right)$ & $0.91\left(R^{2}=0.94\right)$ & & $0.84\left(R^{2}=0.96\right)$ & $0.88\left(R^{2}=0.98\right)$ \\
3 & $0.97\left(R^{2}=0.95\right)$ & $0.94\left(R^{2}=0.93\right)$ & & $0.87\left(R^{2}=0.96\right)$ & $0.96\left(R^{2}=0.99\right)$ \\
\hline
\end{tabular}

Paired $T$ tests were used to determine which variables among $(R, H, k, F)$ were influential on the ratio $Q^{\text {ose }} / Q^{\mathrm{ms}}$. The influence of $R, H$ and $k$ was studied for $F=0$, which means that the focal plane was in the middle of the tube, where the velocity profile can be used to estimate the flow rate. The analysis led to the following results.

- When $Q^{\text {ose }} / Q^{\mathrm{ms}}$ for a $15 \mu \mathrm{m}$ radius vessel (R15) is compared with that of a $20 \mu \mathrm{m}$ radius vessel (R20), all other factors being equal, the paired $t$ test showed no significant difference $(p=0.1)$.

- Moreover, no significant difference was found between the flow rate ratio obtained with a hematocrit of $20 \%$ and that at a hematocrit of $40 \%(p=0.5)$.

- Similarly, no significant difference $(p=0.3)$ was obtained between the flow rate ratio obtained with a parabolic velocity profile $(k=2)$ and that with a blunted profile $(k=3)$. In contrast, a significant difference $(p<0.001)$ was found between the sharp velocity profile $(k=1.5)$ and the parabolic profile $(k=2)$, meaning that the estimated flow rate was less precise when the profile was sharp, as shown in table 1 . For example, for the $15 \mu \mathrm{m}$ vessel with a hematocrit of $20 \%,\left(Q^{\text {ose }} / Q^{\mathrm{ms}}\right)_{k=1.5}$ was found to be equal to 0.88 for $k=1.5$, whereas $\left(Q^{\text {ose }} / Q^{\mathrm{ms}}\right)_{k=2}$ was found to be equal to 0.92 for $k=2$.

Regarding the distance of the focal plane from the center of the tube, as expected paired $t$ tests showed very clearly the influence of this parameter on the ratio $Q^{\text {ose }} / Q^{\mathrm{ms}}$. This parameter had the greatest influence, as shown in the detailed study that follows.

\subsection{Focus effect on the flow estimation}

It is important to quantify the estimation error as a function of the position of the focal plane because in the case of intravital in vivo study it may be difficult to place the focal plane at the center of vessels, especially because the vessel could move during the breath cycle. Even with rigid tubes in vitro, the precise position of the focal plane may be difficult to adjust.

4.2.1. Focus effect on the in vitro flow estimation. Figure 6 presents the curve $Q^{\text {ive }} / Q^{p}$ (in vitro estimated flow divided by the pump flow) as a function of the focus plane. For these experiments, a $15 \mu \mathrm{m}$ radius glass tube was perfused with $\mathrm{H} 40 \%$ blood with constant flow rates of $1.5 \mu \mathrm{L} \mathrm{h}^{-1}$ and $8 \mu \mathrm{L} \mathrm{h}^{-1}$. Each point is the mean of nine flow estimations ${ }^{7}$ and the error bar is the standard deviation of these measurements. The negative axis represents the position of the focus plane above the middle of the tube, whereas the positive axis is under the middle of the tube. We note that the standard deviation is smaller for the $8 \mu \mathrm{L} \mathrm{h}^{-1}$ experiment.

7 A few individual data differing from the average by more than 2 SD have been discarded. 


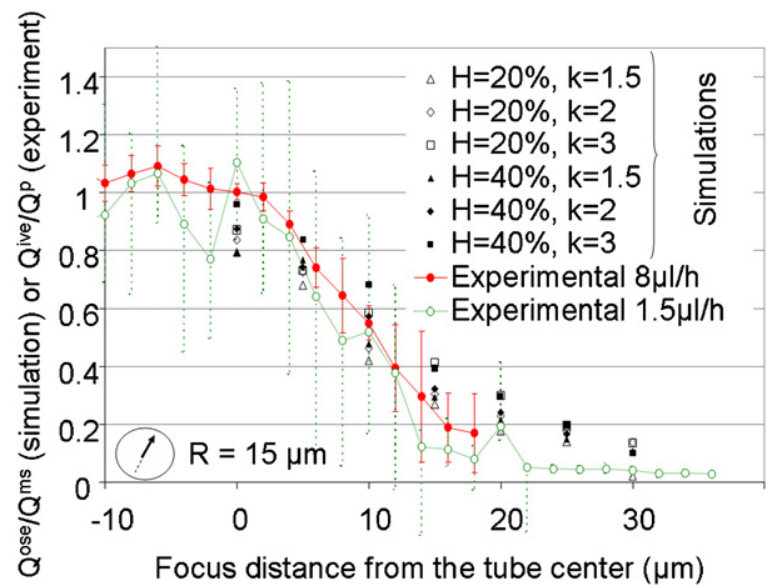

Figure 6. In vitro and in silico estimated flow rates by CC analysis normalized by the gold standard flow (flow rate of the mechanical model or pump flow rate) as a function of the focus plane. For both, tube radii were equal to $15 \mu \mathrm{m}$. For the experiments, the glass tube was perfused with $\mathrm{H} 40 \%$ blood at constant flow rates of 1.5 and $8 \mu \mathrm{L} \mathrm{h}^{-1}$. For in silico, note that each dot is an average for flow rates from 0 to $2 \mu \mathrm{L} \mathrm{h}^{-1}$.

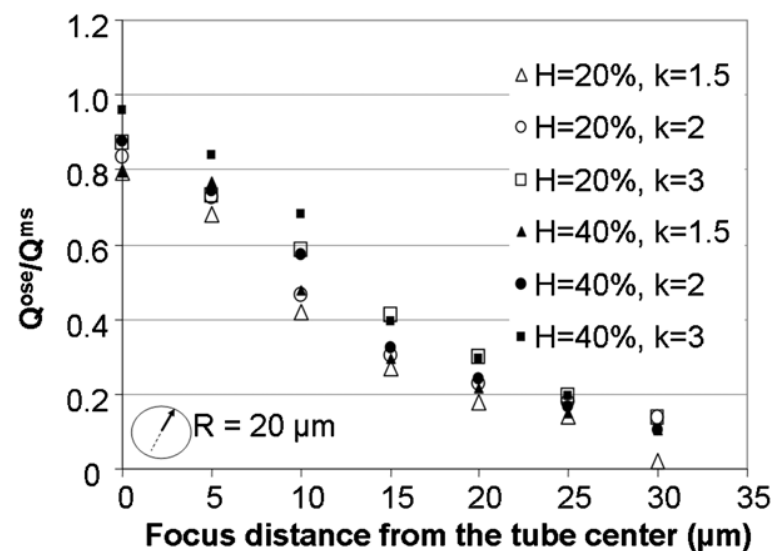

Figure 7. In silico estimated flow rates by CC analysis normalized by the gold standard flow (flow rate of the mechanical model) as a function of the focus plane. Tube radii equal to $20 \mu \mathrm{m}$. Note that each dot is an average for flow rates from 0 to $2 \mu \mathrm{L} \mathrm{h}^{-1}$.

4.2.2. Focus effect on the in silico flow estimation. Figures 6 and 7 show the in silico ratio $Q^{\text {ose }} / Q^{\mathrm{ms}}$ as a function of the focal plane position in simulated vessels with radii of 15 and $20 \mu \mathrm{m}$, respectively. The results from synthetic images are consistent with the experimental results in figure 6 for the $15 \mu \mathrm{m}$ radius glass tube. We notice a shift of the in vitro curve under the middle of the tube that could be due to the high opacity of the blood at $40 \%$ hematocrit, which was not taken into account in the mechanical model. It is clear that when the focal plane was far from the center of the tube, the flow rate was highly underestimated. When the focal plane was located at the center of the simulated tube, the flow rate calculated by the CC method reflected at least $90 \%$ of the gold standard flow rate; the $\mathrm{CC}$ flow was underestimated by $20 \%$ when the focal plane was located $5 \mu \mathrm{m}$ from the center of the tube and by $60 \%$ when 

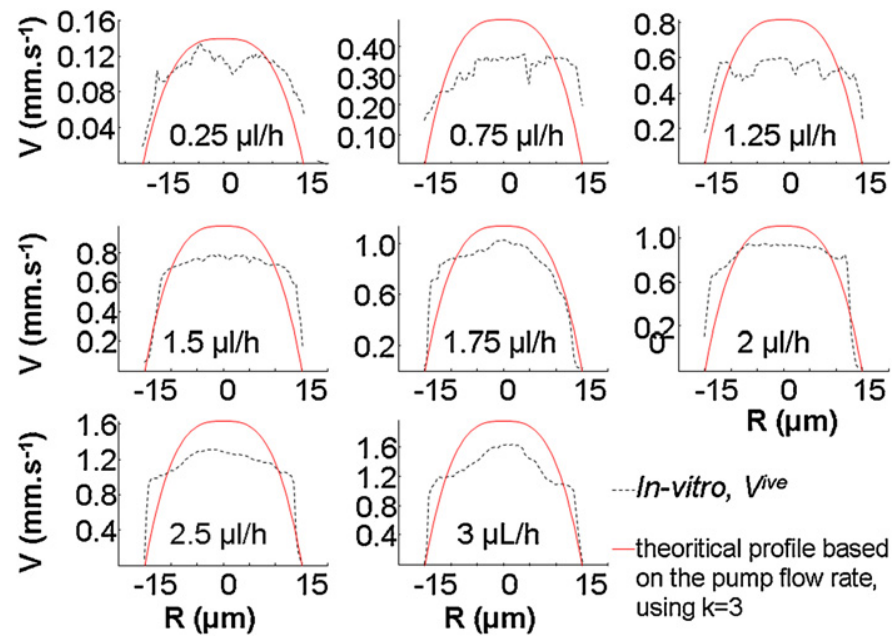

Figure 8. Typical velocity profiles obtained by the $\mathrm{CC}$ method in the in vitro model ( $\left.V^{\mathrm{ive}}\right), H=$ $40 \%$. The continuous red line is for the theoretical velocity blunt profile $(k=3)$ computed based on the pump flow rate.

Table 2. Average $k$ coefficients of the velocity profile shape obtained using CC on the in silico flow compared with the $k$ implemented in the corresponding mechanical model $(k=1.5,2$ and 3$)$

\begin{tabular}{llll}
\hline$k$ & 1.5 & 2 & 3 \\
\hline R15 H20 & $2.2 \pm 0.4$ & $3.0 \pm 1.1$ & $4.3 \pm 1.5$ \\
R15 H40 & $1.6 \pm 0.3$ & $3.1 \pm 1.0$ & $4.6 \pm 1.9$ \\
R20 H20 & $1.4 \pm 0.2$ & $1.8 \pm 0.4$ & $2.9 \pm 1.0$ \\
R20 H40 & $1.3 \pm 0.1$ & $1.8 \pm 0.3$ & $2.8 \pm 0.6$ \\
\hline
\end{tabular}

the focal plane was located $15 \mu \mathrm{m}$ from the center of the tube. At $30 \mu \mathrm{m}$ from the center, the flow rate error was around $90 \%$.

\subsection{Shape of the velocity profile}

The shape of the velocity profile under physiological conditions, which impacts the wall shear rate, is known to depend on the RBC aggregation level and hemodynamic conditions, such as vessel curvature, entrance length, etc (Bishop et al 2001a, 2001b, 2001c). Only data with focus in the middle of the tube are considered here $(F=0)$. Figure 8 (experimental) and figures 9 and 10 (in silico) show examples of velocity profiles obtained using the $\mathrm{CC}$ method. Experimental velocity profiles are compared with a non-Newtonian blunt profile $(k=3)$ computed based on the pump rate. In silico velocity profiles are compared with the corresponding velocity profile imposed in the mechanical model. We note clearly that $\mathrm{CC}$ velocity profiles are blunted due to the averaging effect of the method in the $15 \mu \mathrm{m}$ radius vessel. It is also clear from an observation of these profiles that, even if we can have a reasonable estimation of the flow rate with the $\mathrm{CC}$ method (if the focus plane is centered), the shape of the velocity profile for the radius of $15 \mu \mathrm{m}$ does not reflect the true profile. However, the prediction of the velocity shape is much better for the vessel with a radius of $20 \mu \mathrm{m}$. Table 2, which shows the relation between the shape parameter $k$ obtained from the CC profile and the gold standard coming from the mechanical model, confirms these observations. 

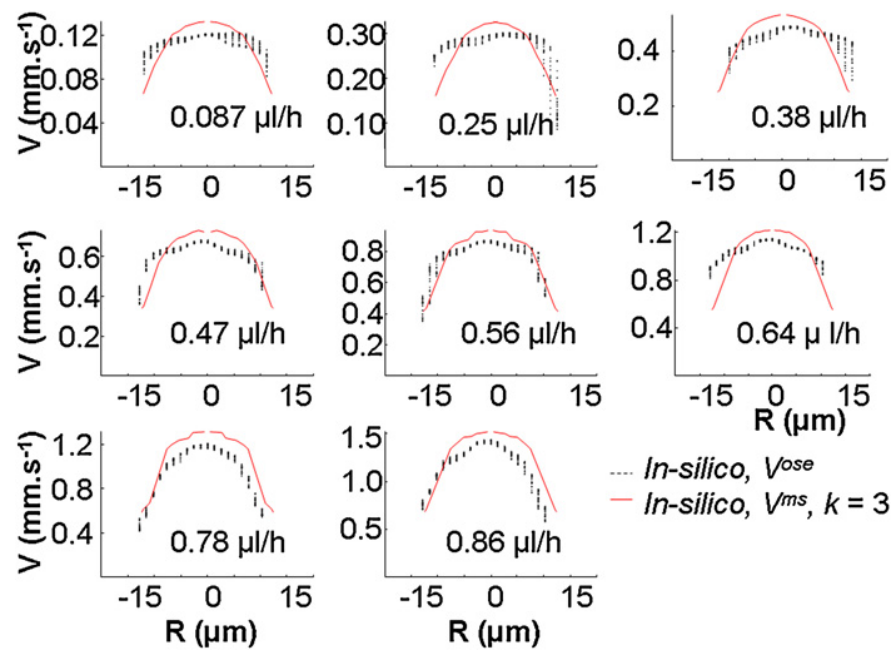

Figure 9. Typical velocity profiles obtained by the $\mathrm{CC}$ method in the in silico model ( $V^{\text {ose }}$ ) for a radius of $15 \mu \mathrm{m}, H=20, k=3$. The continuous line is the $3 \mathrm{D}$ reference velocity profile of the $\mathrm{RBCs}$ in the mechanical model $\left(V^{\mathrm{ms}}\right) . x$-axis: distance from the middle of the tube in $\mu \mathrm{m} ; y$-axis: velocity in $\mathrm{mm} \mathrm{s}^{-1}$.
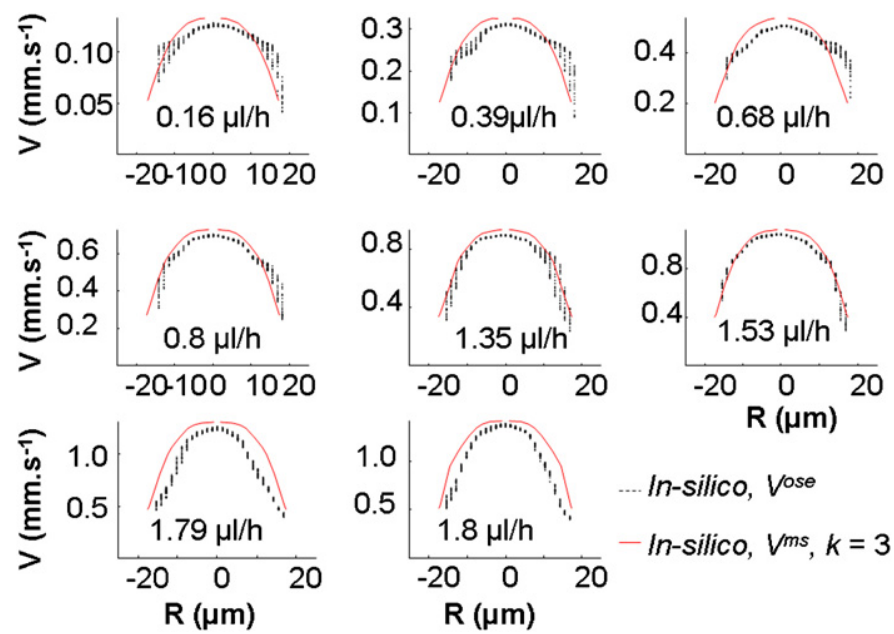

Figure 10. Same as figure 10, but for a radius of $20 \mu \mathrm{m}$.

\section{Discussion}

According to both in vitro and in silico studies, the CC method applied on whole blood optical pictures, in the middle of the tube, gave an estimation of the flow rate with a small underestimation of about $\pm 10 \%$ (for in vitro measurements). As expected, the in silico studies showed smaller underestimations when the velocity profiles are flatter. However, when the focus is not in the middle of the vessel, the underestimation increased drastically with increasing distance from the middle of the tube. For example, the error on the CC flow estimation was two times bigger, when the focal plane was located only $5 \mu \mathrm{m}$ from the center 
of the vessel. On the other hand, the result in the region nearest to the center, at $\pm 2 \mu \mathrm{m}$, showed a stable precision of around $10 \%$. As mentioned earlier, it is difficult to place the focal plane exactly at the center of the vessel, which is why we think that this observation is helpful because the center plane of the tube can more easily be reached with a precision of $\pm 2 \mu \mathrm{m}$. Thus, we recommend, as done by Kloosterman et al (2011), to scan the velocity across the tube and to define the center of the tube as the location where the velocity is maximum.

The CC method on whole blood should not be used to deduce fluid velocity profile shapes for vessels with a radius of $15 \mu \mathrm{m}$ or less, as shown in experimental and in silico data analyses. Despite the theoretical value of the DOC $(14 \mu \mathrm{m})$ computed for the current experimental conditions, in silico data suggest that for the $20 \mu \mathrm{m}$ radius tube, the CC method could provide a better estimation of the velocity profile shape than for the $15 \mu \mathrm{m}$ vessel.

Note that we were able to see a lack of RBC near the wall, a well know phenomena in blood microcirculation called the 'cell free layer'. This phenomenon is due to the spinning of the RBC, making them move toward the center of the tube. We can see it change in the videos as $Q$ changes, but it would be difficult to measure at the magnification we used. In the numerical model, we also observe a smaller density of cells close to the wall due to the elastic interaction forces between the wall and the RBCs. It is also difficult to quantify the thickness of this layer here. This lack of particles close to the wall may affect the accuracy of the cross correlation method close to the wall.

We showed in this study that the use of RBCs as tracer particles in $\mu \mathrm{PIV}$ applications gave biased velocity profiles for vessel diameters corresponding to microcirculatory flow. To circumvent this limitation, several techniques have been adopted to overcome the optical DOC limitation, principally for vessel radii of $50 \mu \mathrm{m}$ and above. Bitsch et al (2005) proposed to use a so-called base-clipping technique. A specific grayscale threshold level was chosen to remove out-of-focus particles and grayscale values below this level were discarded when processing moving speckle in optical images. This approach seems attractive because it is possible to work under physiological conditions with whole blood; its performance, however, has not yet been assessed. Another proposed technique is to label a given proportion of RBCs with florescence to attenuate the effect of concentrated cells, but this technique does not totally eliminate the impact of the DOC. The solution is to sort particles to keep only those in the focal plane by considering the size of the fluorescent spot as done by Bishop et al (2001a) or that of the emitted light intensity as done by Sugii et al (2005). Finally, a promising technique is to add small artificial fluorescent particles, such as proposed by Sugii et al (2005) who computed velocity profiles by adding spherical particles of $1 \mu \mathrm{m}$ diameter using a laser to fluoresce particles in the PIV system. Lima et al (2006, 2007, 2008), also adding $1 \mu \mathrm{m}$ diameter fluorescent microparticles to RBCs, obtained successfully 3D velocities of blood flow in square microchannels. However, to be able to get accurate velocity profiles, the blood had to be diluted to non-physiological hematocrits in order to see fluorescent microparticles. Indeed, the reliability of the velocity profile decreases with the increase of the hematocrit (Lima et al 2007).

We had shown in this study that $\mu \mathrm{PIV}$ using RBCs as tracer particles gave flatter velocity profiles than expected due to the large size of RBCs with respect to the size of the vessel. In agreement with our conclusion, velocity profiles based on RBC tracking were always flattened when compared to detecting RBCs with added fluorescent microparticles. Figure 11 shows normalized velocity profiles extracted from different studies. Even if those profiles were not computed under the same conditions (i.e. vessel diameters, flow rates and hematocrits) the general appearance is specific to the post processing method. Also, we should be careful in such comparisons because results obtained by using artificial fluorescent particles give the velocity of the plasma (or of the surrounding fluid), while methods tracking RBCs focus 


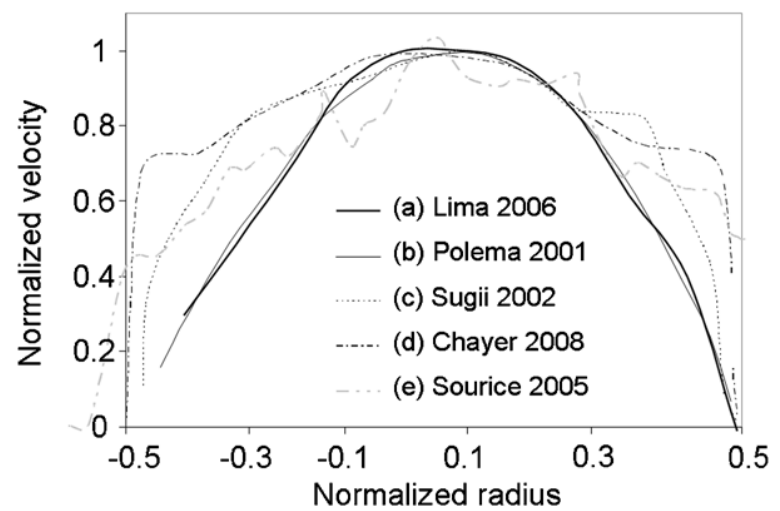

Figure 11. Normalized velocity profiles of microbloodflow extracted from different studies using additional fluorecent particles or not. Continuous lines are used when fluorescent particles are used as tracer particles, dashed lines when RBCs are used as tracer particles. Maximum velocities and vessel diameters are indicated in brackets. (a) Lima et al (2006) blood cell suspension, at $10 \%$ Hct in vitro + fluorescent particle $1 \mu \mathrm{m}\left(0.55 \mathrm{~mm} \mathrm{~s}^{-1}, 100 \mu \mathrm{m}\right)$ (b) Poelma et al (2010), embryonic chicken, blood + fluorecent particle $1 \mu \mathrm{m}\left(40 \mathrm{~mm} \mathrm{~s}^{-1}, 300 \mu \mathrm{m}\right)$ (c) Sugii et al (2002), intravital, embryonic chicken $\left(2.25 \mathrm{~m} \mathrm{~s}^{-1}, 25 \mu \mathrm{m}\right)(\mathrm{d})$ Chayer et al (2008), in vitro circular, blood cell suspension at $20 \% \mathrm{Hct}$, in vitro $\left(11 \mathrm{~mm} \mathrm{~s}^{-1}, 30 \mu \mathrm{m}\right)$ (e) Sourice et al (2005), intravital $\left(0.07 \mathrm{~m} \mathrm{~s}^{-1}, 40 \mu \mathrm{m}\right)$.

on the cell velocity. The comparison of the flow methods deserves to be done in the same experimental conditions.

It should be noted that optical theories on particle image formation have been formulated for the measurement of small, weakly concentrated particles. The in silico model presented in this study can certainly be improved with a more accurate description of the image formation by considering theories on light ray propagation in matter. Moreover, future advancements in the domain of mechanical modeling of flowing RBCs should be profitable, especially to take into account the RBC biconcave shape, RBC deformation and RBC aggregation in the microcirculation.

\section{Conclusion}

We evaluated a tool to quantify velocity profiles measured by optical microscopy when the use of artificial fluorescent particles is not feasible. We have proposed several recommendations for the use of this method.

To summarize, the quantification of the flow rate and velocity profile obtained by the CC method using whole blood has to be interpreted by taking into account the following issues:

- the diameter of the vessel;

- the shape of the velocity profile; and

- the large dispersion of results because of intra and inter blood measurement variabilities.

The original model used to generate synthetic images of optical $\mu$ PIV will support the calibration of post processing methods and the interpretation of in vitro and in vivo data. The database of the 1400 movies can be used by the community on request. The method is also presented in complete detail to be easily adapted for all vessel shapes, sizes, velocity profiles and concentrations of red blood cells. 


\section{Acknowledgments}

This research was supported by the Natural Sciences and Engineering Research Council of Canada. We are indebted to the 'Réseau Québécois de Calcul de Haute performance' (RQCHP) for the generous allocation of computer resources.

\section{References}

Bishop J J, Nance P R, Popel A S, Intaglietta M and Johnson P C 2001a Effect of erythrocyte aggregation on velocity profiles in venules Am. J. Physiol. Heart Circ. Physiol. 280 H222-36

Bishop J J, Popel A S, Intaglietta M and Johnson P C 2001b Rheological effects of red blood cell aggregation in the venous network: a review of recent studies Biorheology 38 263-74

Bishop J J, Popel A S, Intaglietta M and Johnson P C 2001c Effects of erythrocyte aggregation and venous network geometry on red blood cell axial migration Am. J. Physiol. Heart Circ. Physiol. 281 H939-50

Bitsch L, Olesen L H, Westergaard C H, Bruus H, Klank H and Kutter J P 2005 Micro particle-image velocimetry of bead suspensions and blood flows Exp. Fluids 39 507-13

Chayer B, de Guise J A and Cloutier G 2008 Effect of depth of correlation on cross-correlation blood flow measurements in glass microchannels 2008 IEEE Int. Symp. on Biomedical Imaging: from Nano to Macro vols $1-4$ pp 1359-62

Cheezum M K, Walker W F and Guilford W H 2001 Quantitative comparison of algorithms for tracking single fluorescent particles Biophys. J. 81 2378-88

Duran J 1997 Modélisations numériques Sables, Poudres Et Grains: Introduction à La Physique Des Milieux Granulaires ed P Eyrolles (Paris: Impr. Jouve) pp 215-26

Fenech M, Chayer B and Cloutier G 2008 Synthetic images of blood microcirculation to assess precision of velocity profiles by a cross-correlation method IEEE Int. Symp. on Biomedical Imaging: from Nano to Macro vols 1-4 pp 153-6

Fenech M, Garcia D, Meiselman H J and Cloutier G 2009 A particle dynamic model of red blood cell aggregation kinetics Ann. Biomed. Eng. 37 2299-309

Kikuchi K and Mochizuki O 2011 Micro-PIV (micro particle image velocimetry) visualization of red blood cells (RBCs) sucked by a female mosquito Meas. Sci. Technol. 22064002

Kloosterman A, Poelma C and Westerweel J 2011 Flow rate estimation in large depth-of-field micro-PIV Exp. Fluids $\mathbf{5 0} 1587-99$

Koutsiaris A G and Pogiatzi A 2004 Velocity pulse measurements in the mesenteric arterioles of rabbits Physiol. Meas. 25 15-25

Lee J Y, Ji H S and Lee S J 2007 Micro-PIV measurements of blood flow in extraembryonic blood vessels of chicken embryos Physiol. Meas. 28 1149-62

Lima R, Wada S, Takeda M, Tsubota K and Yamaguchi T 2007 In vitro confocal micro-PIV measurements of blood flow in a square microchannel: the effect of the haematocrit on instantaneous velocity profiles J. Biomech. 40 2752-7

Lima R, Wada S, Tanaka S, Takeda M, Ishikawa T, Tsubota K, Imai Y and Yamaguchi T 2008 In vitro blood flow in a rectangular PDMS microchannel: experimental observations using a confocal micro-PIV system Biomed. Microdevices $10153-67$

Lima R, Wada S, Tsubota K and Yamaguchi T 2006 Confocal micro-PIV measurements of three-dimensional profiles of cell suspension flow in a square microchannel Meas. Sci. Technol. 17 797-808

Meinhart C D, Wereley S T and Gray M H B 2000 Volume illumination for two-dimensional particle image velocimetry Meas. Sci. Technol. 11 809-14

Tuma R F, Duran W L and Ley K (ed) 2008 Handbook of Physiology: Microcirculation (New York: Academic)

Nguyen C V, Fouras A and Carberry J 2010 Improvement of measurement accuracy in micro PIV by image overlapping Exp. Fluids 49 701-12

Olsen M G and Adrian R J 2000 Out-of-focus effects on particle image visibility and correlation in microscopic particle image velocimetry Exp. Fluids 29 S166-74

Poelma C, Van der Heiden K, Hierck B P, Poelmann R E and Westerweel J 2010 Measurements of the wall shear stress distribution in the outflow tract of an embryonic chicken heart J. R. Soc. Interface 7 91-103

Poelma C, Vennemann P, Lindken R and Westerweel J 2008 In vivo blood flow and wall shear stress measurements in the vitelline network Exp. Fluids 45 703-13

Popel S and Johnson P C 2005 Microcirculation and hemorheology Annu. Rev. Fluid Mech. 37 43-69

Sourice A, Plantier G and Saumet J 2005 Red blood cell velocity estimation in microvessels using the spatiotemporal autocorrelation Meas. Sci. Technol 162229 
Sugii Y, Nishio S and Okamoto K 2002 In vivo PIV measurement of red blood cell velocity field in microvessels considering mesentery motion Physiol. Meas. 23 403-16

Sugii Y, Okuda R, Okamoto K and Madarame H 2005 Velocity measurement of both red blood cells and plasma of in vitro blood flow using high-speed micro PIV technique Meas. Sci. Technol. 16 1126-30

Tsukada K, Minamitani H, Sekizuka E and Oshio C 2000 Image correlation method for measuring blood flow velocity in microcirculation: correlation 'window' simulation and in vivo image analysis Physiol. Meas. 21 459-71

Vennemann P, Kiger K T, Lindken R, Groenendijk B C W, Stekelenburg-de Vos S, ten Hagen T L M, Ursem N T C, Poelmann R E, Westerweel J and Hierck B P 2006 In vivo micro particle image velocimetry measurements of blood-plasma in the embryonic avian heart J. Biomech. 39 1191-200

Wereley S T, Santiago J G, Chiu R, Meinhart C D and Adrian R J 1998 Micro-resolution particle image velocimetry Proc. SPIE 3258 122-33 